\title{
Diseño de un blog para la divulgación de nanociencia y nanotecnología
}

\author{
Lady Johana Torres Romero y Leidy Bibiana Ariza Traslaviña
}

\begin{abstract}
Resumen
Se presenta el diseño y creación de un blog con el fin de exponer materiales educativos recopilados a partir de diferentes experiencias, relacionadas con la enseñanza de nanociencia y nanotecnología, implementadas en colegios de educación formal en Bogotá, Colombia. Esto, a partir de estrategias como juegos de roles, salidas de campo, prácticas de laboratorio y otros materiales didácticos. Este trabajo nace con el diseño de una propuesta de alfabetización científica en los temas y estructuras de escala nano. Dicha propuesta también habrá de permitir la articulación con temas comunes, pertenecientes al plan de estudios de la educación básica y media en Colombia. Posterior a la aplicación de esta propuesta, surgen diferentes modificaciones en relación con el nivel de estudios y las necesidades educativas, que se socializan mediante este blog. Por esta razón, se exponen algunas experiencias obtenidas a raíz de este contexto, con el fin de servir como referencia y motivación para otros investigadores especializados en el área, pues se trata de un campo poco explorado en el país. A partir de esta experiencia inicial, se reconoce la importancia de indagar más en esta temática, que les permite a los estudiantes de educación formal y no formal fortalecer diferentes competencias y habilidades, como la toma de decisiones y pensamiento crítico acordes con su contexto y la relación con las ciencias.
\end{abstract}

Palabras clave: enseñanza, nanociencia, nanotecnología, blog, difusión, experiencias educativas, educación secundaria.

\section{DESIGN OF A BLOG fOR THE DISSEMINATION OF NANOSCIENCE AND NANOTECHNOLOGY}

\begin{abstract}
The design and creation of a blog is presented here in order to expose educational materials compiled from different experiences related to teaching nanoscience and nanotechnology. Such experiences have been implemented in formal education schools in Bogotá (Colombia) using strategies such as role plays, field trips, laboratory practices and other teaching materials. This work originally conceived as scientific literacy project proposal that would allow the articulation of common themes belonging to the basic and secondary education curriculum with nano themes and structures. After the application of this proposal, different modifications, according to the level of study and the educational needs, are socialized through this blog, so that they can serve as a reference and motivation for other researchers in this area barely explored in Colombia. From the initial experience, the importance of investigating more in this subject is recognized, which allows students of formal and non-formal education to strengthen different competences and skills, such as decision-making and critical thinking, according to their context and relationship with science.
\end{abstract}

Keywords: teaching, nanoscience, nanotechnology, blog, diffusion, educational experiences, secondary education.

Recepción: 15/09/2019. Aprobación: 27/07/2020.

Dol: http://doi.org/10.22201/cuaieed.16076079e.2020.21.6.10 
"Diseño de un blog para la divulgación de nanociencia y nanotecnología" Lady Johana Torres Romero y Leidy Bibiana Ariza Traslaviña Vol. 21, Núm. 6, noviembre-diciembre 2020 Revista Digital Universitaria

Lady Johana Torres Romero

leidyto_4@hotmail.com orcid.org/0000-0002-7900-9272

Magister en enseñanza de las ciencias exactas y naturales de la Universidad Nacional de Colombia. Licenciada en Química de la Universidad Pedagógica Nacional. Experiencia como asesora pedagógica, profesional en manejo de laboratorios, y docente de química, biología, ciencias naturales, ciencia y tecnología, y nanotecnología, en diferentes niveles educativos, con niños, jóvenes, adultos y docentes. Manejo de población vulnerable empleando modelos educativos flexibles (MEF).

Colaboradora en el grupo de investigación Nuevos Materiales Nano y Supramoleculares de la Universidad Nacional de Colombia, y en el grupo de investigación en educación y divulgación de la nanociencia y la nanotecnología EDUDINA de la Academia Colombiana de Ciencias exactas, físicas y naturales.

\section{Leidy Bibiana Ariza Traslaviña}

arizabibiana@gmail.com

Licenciada en química y Magister en Docencia de las Ciencias Naturales de la universidad pedagógica nacional, experiencia como docente de biología, química y física en básica secundaria y educación media, investigadora asociada en historia y epistemología de las ciencias, grupo Ecoperspectivas, trabajo experimental en el grupo de calorimetría y sólidos porosos de la universidad de los Andes en colaboración con la Universidad Pedagógica Nacional. Asesora externa en Proyecto Educativo Institucional (PEI) y sistemas de evaluación en Colombia.

\section{Introducción}

Desde hace algunos años han surgido líneas de investigación y corrientes didácticas que se enfocan en informar sobre las problemáticas ambientales actuales, y que también son usadas como mecanismos generadores de atención, motivación y aprendizaje por las ciencias naturales. Entre estas tendencias, podemos mencionar temas como la química verde, agricultura urbana, gastronomía molecular, cambio climático, energías alternativas, robótica, biotecnología, nanociencia y nanotecnología.

Cuando se vinculó esta variedad de campos disciplinarios con las experiencias propias de niños y jóvenes, se detectó que estos grupos muestran un particular interés por la nanociencia y la nanotecnología. A pesar de que ya se había dejado entrever el quehacer de estas áreas científicas desde la conceptualización del átomo, el público en general dimensiona con dificultad la variedad de fenómenos naturales y desarrollos tecnocientíficos que nuestra juventud puede usar a su favor. En este sentido, la enseñanza de nanociencia y nanotecnología en la educación secundaria de Colombia aún es incipiente. Además, debido a su inherente complejidad, se considera que no deberían enseñarse estos temas a la población juvenil e infantil en la educación formal, pues no están contemplados en los estándares de competencias curriculares del Ministerio de Educación Nacional (MEN, Torres y Duarte, 2018). 
"Diseño de un blog para la divulgación de nanociencia y nanotecnología" Lady Johana Torres Romero y Leidy Bibiana Ariza Traslaviña Vol. 21, Núm. 6, noviembre-diciembre 2020 Revista Digital Universitaria

Por esta razón los docentes de ciencia, quienes a su vez asumen el rol de comunicadores, deben utilizar diferentes recursos y medios para informar y fomentar en sus alumnos el pensamiento y razonamiento científicos. De esta forma, se cubren contextos, no sólo escolares, sino también aquellos en los que cualquier persona, sin importar su formación, pueda familiarizarse sobre temas como nanociencia y nanotecnología.

La importancia de la educación en nanotecnología nos lleva a los docentes a repensar nuestra práctica, para así considerar la inclusión de estos temas en el aula y, sobre todo, contribuir a la formación de ciudadanos que se desenvuelvan en una era tecnológica, aunque no se vea simple vista, cada vez más inmersa en los artículos nano.

El prefijo nano- se refiere a, por una parte, los fundamentos, propiedades y diseño de objetos (nanociencia) y, por la otra, a dispositivos con funciones específicas (nanotecnología), cuyas dimensiones son de unos pocos nanómetros. El nanómetro $(\mathrm{nm})$ es una unidad de longitud equivalente a la millonésima parte de un milímetro (10-9 m) (Serena Domingo, 2002).

De acuerdo con lo anterior, dentro de la enseñanza de las ciencias en la educación básica y media, han surgido profesionales e instituciones que, aunque de forma incipiente, han trabajado para llevar los temas nano a las aulas. No obstante, falta mayor divulgación para generar antecedentes importantes en este campo y aumentar el número de investigadores motivados a trabajar en él.

En el trabajo de Rubiano (2013), se realizó el diseño de una unidad didáctica denominada Viaje al interior de los objetos: el fantástico mundo de lo diminuto, dirigida a docentes, con el objetivo de que los estudiantes se aproximen a algunos conceptos de nanociencia y nanotecnología. Por otro lado, se diseñó un material educativo para la enseñanza de los nanomateriales, por parte de Rubiano (2015), denominado Nanobox, el cual desarrolla, por ejemplo, consecuencias de la escala nano o propiedades eléctricas y físicas de los nanomateriales.

Este recorrido por algunas experiencias docentes permite la socialización de la nanotecnología, pues nos dejó ver lo interesante de cada una de las propuestas. Por esta razón, se acude a un medio de alto impacto, como internet, donde sea posible generar redes de interacción social para crear alianzas, establecer redes de trabajo y de cooperación entre estos investigadores e instituciones, con la posibilidad de promover experiencias y proyectos. Así surge Nanotecnología en la Educación Secundaria en Colombia, que a su vez funciona como medio de divulgación de información científica que permite llamar la atención de la población en general para acercarse a los proyectos en curso y se fortalezca el conocimiento en este tema que, como ya se mencionó, no ha sido aún muy explorado. 
"Diseño de un blog para la divulgación de nanociencia y nanotecnología"

Lady Johana Torres Romero y Leidy Bibiana Ariza Traslaviña

Vol. 21, Núm. 6, noviembre-diciembre 2020

Revista Digital Universitaria

Desde el año 2014, se empezaron a implementar actividades con estudiantes de ciclo $v_{1}^{1}$ por parte de las docentes investigadoras. La primera intervención se llevó a cabo en el Colegio Rural Douglas Brown de Zipaquirá con 35 estudiantes de décimo grado. Ahí mismo se realizó un trabajo para acercar los contenidos y lenguaje científicos que rodean al tema nano a los estudiantes. Este método de intervención fue asociado a la educación formal y articulado con el currículo de ciencias para el grado correspondiente. Este trabajo se convirtió en una estrategia que promovería la alfabetización científica. Para ello, se propuso crear un glosario nanotecnológico con todo el grupo de estudiantes, quienes tenían el reto de consultar los términos y buscar una imagen que sirviera para explicarle a sus compañeros lo que habían entendido al respecto. Se repartieron diferentes términos en el grupo, tales como el de nanómetro, nanotecnología, nanotubo o nanociencia. También se involucraron términos propios de la química y se realizaron prácticas experimentales con materiales como aerogeles de carbono y carbones activados.

Éstas y otras estrategias implementadas por las docentes, en diferentes instituciones educativas en Bogotá, son compartidas en el blog, no sólo como una contribución a la educación formal, sino también a la educación no formal. Precisamente, se decidió la creación del blog para la difusión de lo que, como docentes investigadoras, hemos realizado y lo que otros han hecho. Así, publicar experiencias y proyectos de instituciones, profesionales e investigadores (a quienes se les invita a compartirnos sus experiencias), tiene el fin de conformar una página de consulta e investigación con posibilidades de balancear el bajo índice de divulgación y enseñanza de la nanotecnología, en la educación secundaria en Colombia.

En este artículo se encontrarán aspectos teóricos sobre el blog, la divulgación de la nanotecnología, metodología, resultados, conclusiones y bibliografía.

\section{El blog}

Entre las tecnologías y herramientas web 2.0 al alcance de la educación, destacan los blogs. Según la definición del lingüista José Antonio Millán, un blog es "una página web que consta de entradas (o posts) en orden cronológico inverso, compuestas por texto, imágenes o incluso videos y audios, que normalmente permite a los visitantes dejar comentarios, y que, por último, se realiza mediante algún programa gratuito directamente desde la Web" (2007). Los beneficios que la utilización de los blogs ofrece en el ámbito educativo son diversos por su sencillez, comodidad y facilidad de uso. Los más destacados, según la Junta de Andalucía (citado en Quince, 2016), son:

- Los contenidos multimedia son motivadores y enriquecen el proceso de aprendizaje del olumno.

1 En Colombia corresponde al grado decimo de la educación media.
- Rompen las restricciones de tiempo y espacio, favoreciendo el aprendizaje continuo. 
- Un buen medio para desarrollar la competencia digital.

- Posibilita nuevas formas de comunicación.

De acuerdo con García (2006), los blogs tomaron fuerza y popularidad en 1999 con el surgimiento de Blogger, una empresa estadounidense que ofrece la facilidad de crear y publicar textos con una interfaz fácil, que ha posibilitado a los usuarios formar lazos sociales. Ésta es la principal razón de nuestra actividad, promover los vínculos necesarios para hacer un campo de exploración cada vez más amplio.

El uso más distintivo que se le puede dar a un blog es su aprovechamiento como medio de publicación de resultados de investigación. Con los blogs se eliminan a los intermediarios y desaparece el impasse de espera que conlleva la revisión de los trabajos en las revistas científicas, a la vez que supone una inmejorable forma de hacer llegar la información al resto de la comunidad de una forma más rápida, directa y personal (Torres, y Cabezas, s.f.).

\section{Divulgación de la nanotecnología}

Aunque la difusión y la divulgación están muy vinculadas, tienen diferencias sustanciales. La difusión de la ciencia es una actividad cuyo mensaje apunta a un público especializado, en un determinado tema. La divulgación, por el contrario, busca que el mensaje sea asequible para todo tipo de personas (cITNOVA).

Para fomentar la asimilación de contenidos en nanocienciay nanotecnología debe coexistir la divulgación y la formación. La divulgación hace accesible el conocimiento al público en general, mientras que la formación tiene que ver con la educación formal que se puede presentar en diferentes ciclos o etapas como la educación primaria, secundaria o universitaria (Tutor-Sánchez, 2013). De esta manera,

La divulgación de la nanotecnología constituye un reto de cierta complejidad que es necesario abordar de una manera planificada, desde una perspectiva multidisciplinar y apoyándose en una gran variedad de canales existentes: folletos, guías, libros divulgativos, documentales, debates y programas monográficos de radio y televisión, modelos y maquetas tridimensionales, exposiciones en museos de la ciencia o en museos móviles, videojuegos, concursos, visitas guiadas a centros de investigación, páginas web de contenido educativo o divulgativo, blogs, redes sociales, etc. (Serena, 2013, p. 6).

En la figura 1a se presentan logos de algunas estrategias que han surgido en torno a la preocupación de capacitar y formar a la sociedad a nivel internacional en el área de nanociencia y nanotecnología; y en la figura 1 b instituciones a nivel Colombia que están trabajando en el tema (Torres, 2018). 
Figura 1. Estrategias de divulgación y formación. a. Nivel internacional. b. A nivel Colombia. Fuente: Torres, 2018.

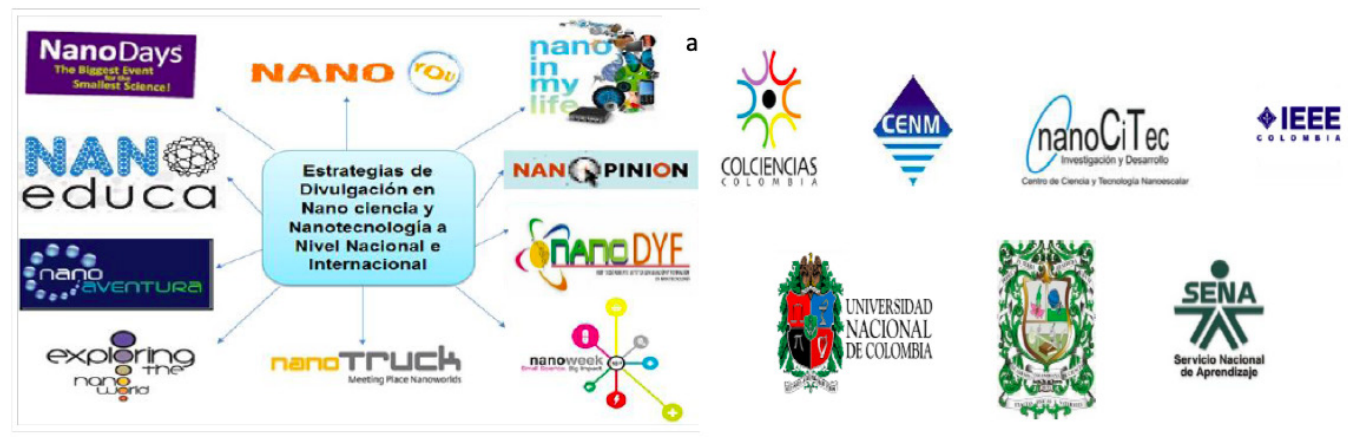

\section{Metodología}

El blog como una propuesta de intervención o creación de contenidos reúne diversas estrategias educativas, empleadas por las docentes investigadoras, sobre diferentes actividades, como juegos de roles, prácticas de laboratorio o salidas pedagógicas. Dichas estrategias y actividades, además de que tienen la intención de aproximar a los estudiantes a temas de nanocienciay nanotecnología, pueden servir también como guía y antecedente para otros docentes o, incluso, para investigación educativa sobre temas relacionados. La metodología es de tipo cualitativo y conlleva seis etapas, las cuales se observan en la figura 2 y se describen a continuación.

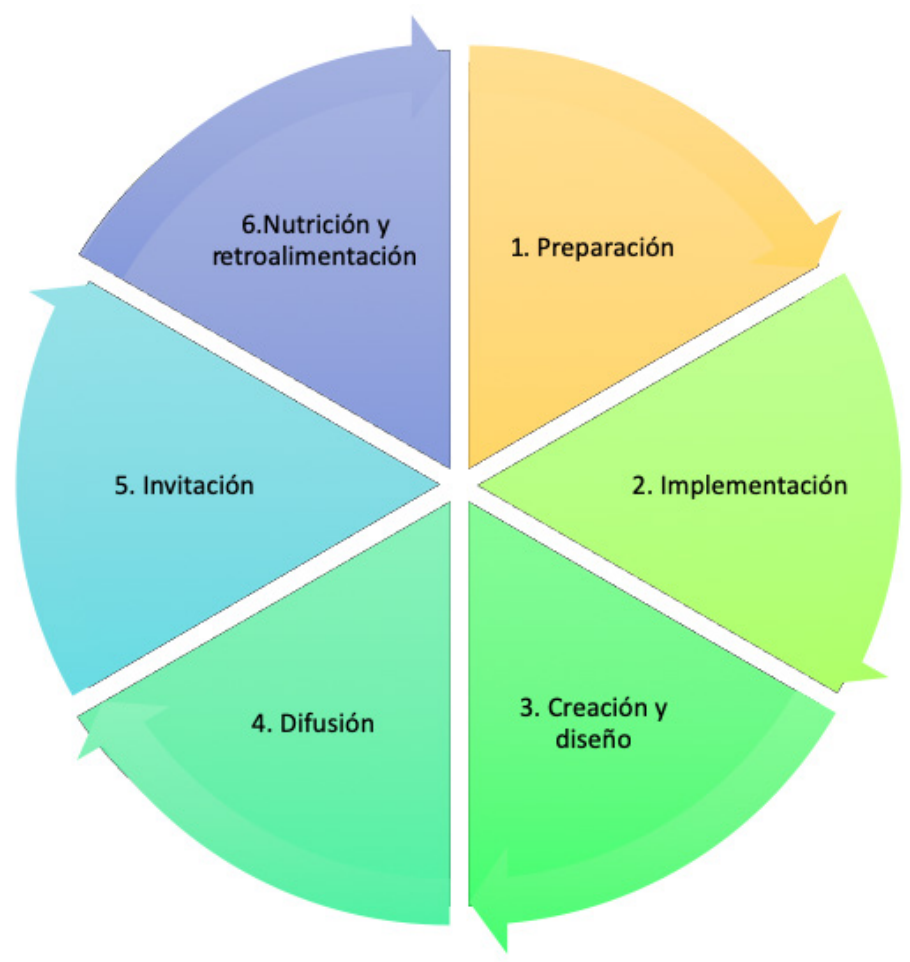

b

\section{E}

Figura 2. Etapas de investigación. 
- Etapa 1: preparación. En esta primera parte se hace una planeación de los contenidos propios de la nanociencia y nanotecnología, que se pueden llegar a vincular e involucrar con temáticas de las ciencias naturales. Se consideran los estándares de competencias curriculares en ciencias del men para el ciclo v (grados 10 y 11).

- Etapa 2: implementación. Una vez concluida la planeación de las clases y materiales didácticos, se involucra a estudiantes de educación media en diversas instituciones de Bogotá, y se emplean diversas estrategias y metodologías para acercarlos a estos contenidos. Entre ellas, se pueden destacar las siguientes: juego de roles, laboratorios, salidas de campo, uso de mapas conceptuales, uso de alimentos, materiales didácticos, etcétera.

- Etapa 3: creación y diseño. Por ser una página de fácil acceso, se realiza el diseño del blog en la plataforma Wordpress y se empiezan a crear las diferentes entradas del blog en torno a las temáticas implementadas. Enseguida, se organizan los contenidos e información relevante de cada experiencia de aprendizaje con los estudiantes y se seleccionan las imágenes resultantes del proceso de ejecución.

- Etapa 4: difusión. Se difunde la propuesta del blog con otros profesionales y se les hace la invitación para compartir experiencias del ámbito nano, para que sean publicados en el sitio

- Etapa 5: invitación. Se publica la página oficialmente en la red y, por medio de varios profesionales, se invita a compartir el blog en diversos grupos de investigación y demás páginas de instituciones y universidades.

- Etapa 6: nutrición y retroalimentación. A medida que se van sumando más experiencias, se nutre la página con estas intervenciones y se hace retroalimentación y análisis de estas aplicaciones. En este punto se empiezan a generar alianzas y convenios.

\section{Resultados}

\section{Preparación e implementación}

Se diseñaron estrategias implementadas en diferentes instituciones de Bogotá. Entre éstas se destacan el colegio rural Douglas Brown de Zipaquirá, la corporación educativa Minuto de Dios, la IED España, la Summerhill School, la Universidad Antonio Nariño, entre otras. Una actividad que se implementó fue denominada "El glosario nanotecnológico", en la cual las docentes asignaron diferentes conceptos de química y nanotecnología a los estudiantes. Éstos debían realizar una explicación, con sus propias palabras, de lo que habían entendido y emplear una imagen como apoyo. En cada clase los estudiantes 
Figura 3. Implementación de "El glosario nanotecnológico". a y b imágenes de apoyo empleadas por los estudiantes. c. Estudiante exponiendo su trabajo. expusieron sus conceptos y las docentes supervisaron y realizaron las correcciones pertinentes, en caso de haber algún malentendido de apropiación del concepto. Este ejercicio fue apropiado, ya que cuando se usaban algunos de estos términos en las clases, los estudiantes ya sabían a qué hacía referencia y, al final del proceso, se observó un progreso en su lenguaje científico. En las figuras $3 a$ y $3 b$ se observan algunas imágenes de los carteles que hacían los estudiantes para sus exposiciones, y en la figura 3c se ve a una de las estudiantes exponiendo su trabajo.
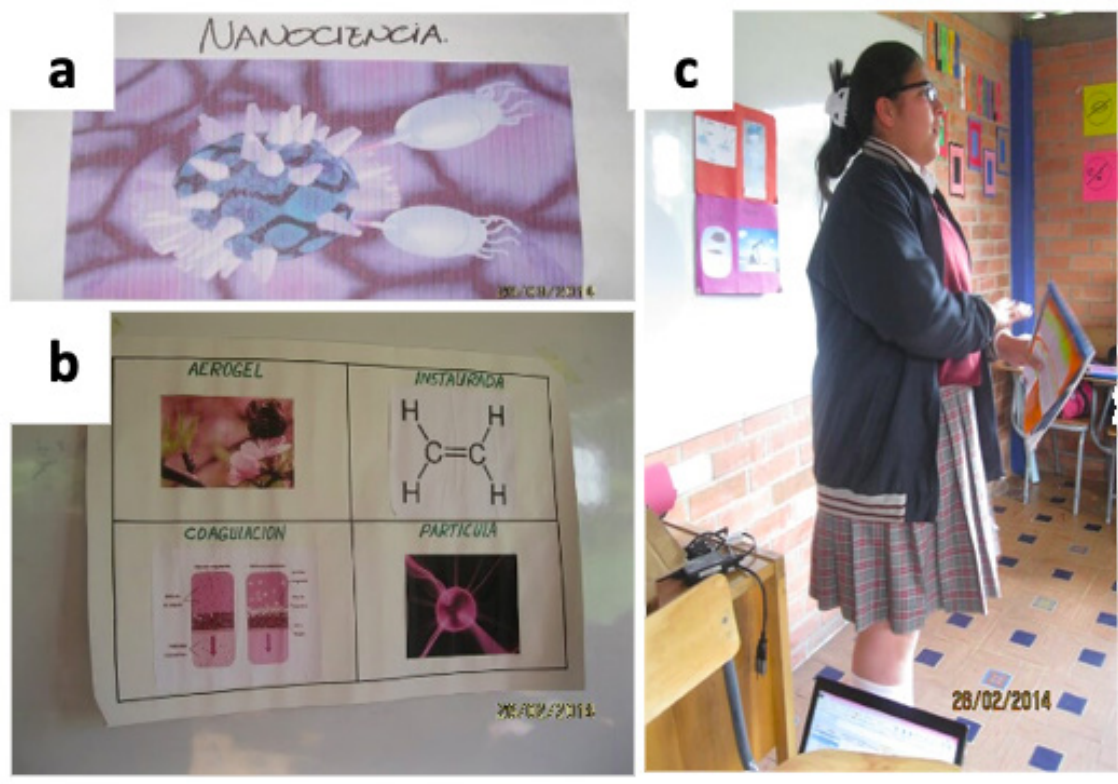

Con este grupo de estudiantes se hicieron otras intervenciones que podrán ser consultadas en el blog Enseñanza de la nanotecnología en la educación secundaria en Colombia.

\section{Creación y Diseño}

Se llevó a cabo la creación y organización de contenidos del blog en la página Wordpress.

En el blog se encuentra la "Página de introducción", en la cual se muestran sus objetivos y finalidades, y se hace la invitación de publicar, comentar y compartir experiencias.

En la parte izquierda están las entradas del blog. Cada una contiene una temática diferente, según las estrategias o actividades implementadas por las docentes investigadoras. Entre las entradas se pueden mencionar las siguientes: Glosario nanotecnológico, Mapas conceptuales y nanotecnología, Naturaleza y nanotecnología, El celular y la nanotecnología, Propiedades físicas y químicas, Juegos de roles, Laboratorio materiales adsorbentes, nanopartículas y alimentos, 
Salida a PTAR, El Salitre, nanopartículas y alimentos, Docente nano, y Proyectos de investigación escolar.

En la figura 4 se puede apreciar la página principal del blog y algunas de sus entradas.

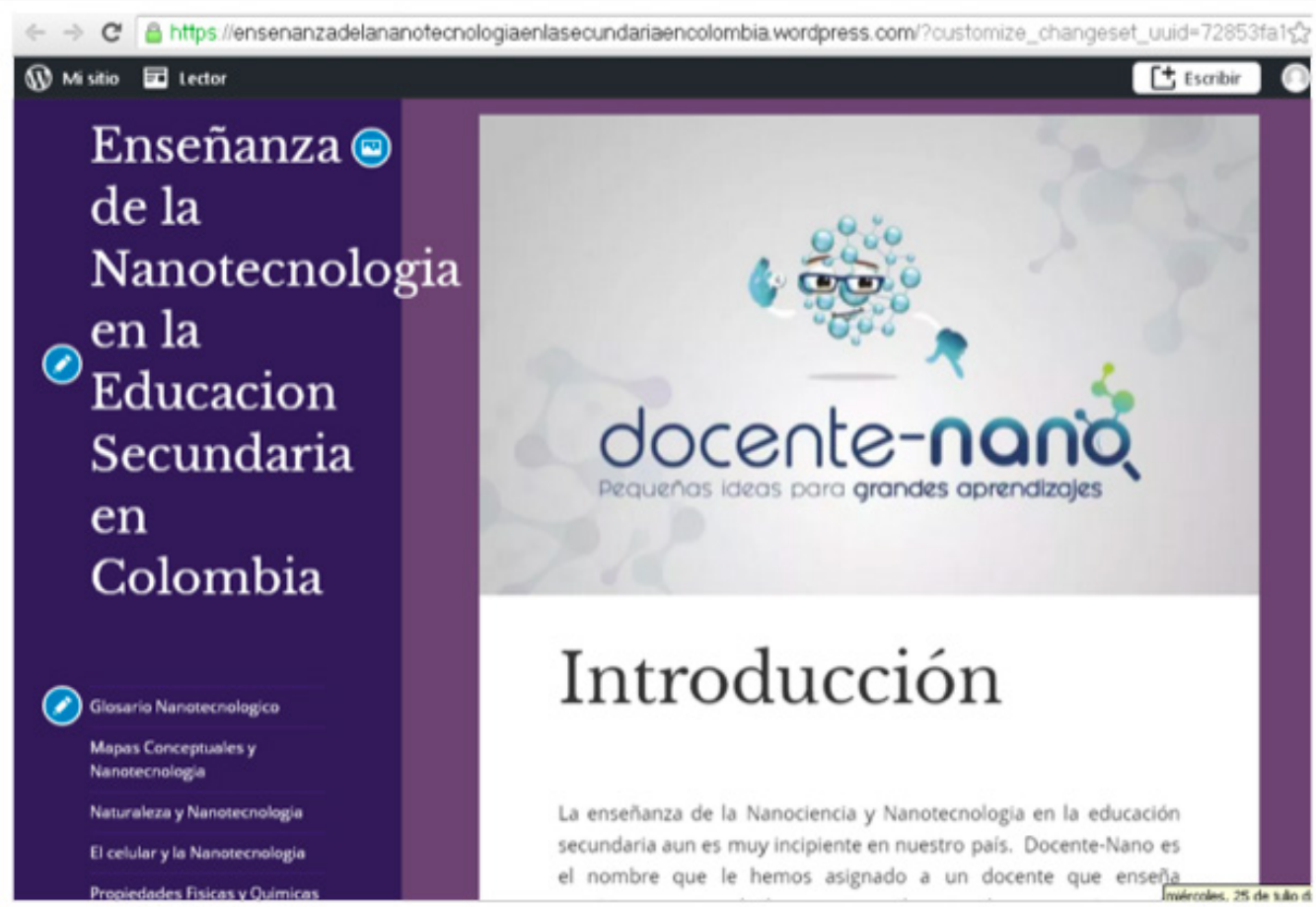

Figura 4. Página inicial del blog Enseñanza de la Nanotecnología en la educación secundaria en Colombia

${ }^{2}$ Es el centro internacional de negocios y exposiciones de Bogotá que promueve la organización de ferias, exposiciones, eventos y convenciones.
Para que haya comunicación con miembros de otras comunidades científicas o académicas, el blog contiene una página denominada "Contactos", en la que se encuentra toda la información de las investigadoras. Posteriormente se irán involucrando otros miembros y profesionales que estén trabajando en el tema, así como quienes nos compartan sus experiencias.

\section{Difusión}

Se hace propagación del blog con otros profesionales e instituciones. Entre ellos, podemos destacar el trabajo realizado con la Universidad Antonio Nariño, en Expociencia 2017. Sobre este trabajo se publicaron cinco entradas del blog, una por cada mesa de trabajo en Corferías, ${ }^{2}$ en el stand denominado "Cacharrear con ciencias: MundoNANO". De esas entradas, se destacan las siguientes: Escala de tamaños ¿Nano qué?, El nanomundo en la naturaleza y su historia, ¿Para qué sirve la nanotecnología?, ¿Cómo hacemos y estudiamos cosas nano?, y Juguemos con nanotecnología. 


\section{Invitación}

Se publica la página con el siguiente enlace: https:// ensenanzadelananotecnologiaenlasecundariaencolombia.wordpress.com/ Gracias a esto, se empieza a generar difusión de las estrategias pedagógicas implementadas con estudiantes de educación secundaria, para la enseñanza de la nanotecnología.

El blog se continuará nutriendo de experiencias de las investigadoras y otros profesionales, pero también se buscará vincular la participación de estudiantes mediante foros, publicación de talleres, ferias de ciencia y demás actividades en los que éstos se vean involucrados.

\section{Conclusiones}

El blog es una herramienta ideal para compartir experiencias pedagógicas y darlas a conocer a una comunidad en específica. En este caso, el blog sobre enseñanza de la nanotecnología en la educación secundaria está dirigido a docentes e investigadores. El blog es empleado como una alternativa para recopilar antecedentes, que puedan ser la base para inspirar y motivar a otros profesionales a asumir el reto de llevar estas temáticas, mediante las experiencias y vivencias que allí se comparten.

Es importante que los docentes publiquen todo lo que hacen $y$, de esta manera, den a conocer su trabajo para que se generen nuevas alianzas, contactos y convenios, para potencializar proyectos en pro de una educación más completa e innovadora en la enseñanza de las ciencias.

\section{Referencias}

* García, L. (2006). Usos y apropiaciones de los blogs de Tijuana Bloguita Front [tesis de maestría, Instituto Tecnológico y de Estudios Superiores de Occidente]. http:// hdl.handle.net/11117/2409

* Junta de Andalucía. (s. f.). Ventajas del uso de los blogs educativos [consultado el 10 de abril de 2015]. http://www.juntadeandalucia.es/averroes/centrostic/14700420/ helvia/aula/archivos/repositorio/0/142/html/ventajas_del_uso_de_blogs_ educativos.htm

* Millán J. A. (2007). Vocabulario de ordenadores e Internet. Jamillan [consultado el 11 de abril de 2015]. http://jamillan.com/v_blog.htm

Quince, A. (2016). El uso del blog en el área de ciencias naturales. Publicaciones Didácticas. http://publicacionesdidacticas.com/hemeroteca/articulo/068076/ articulo-pdf 
* Rubiano, C. (2015). Desarrollo de material educativo en nanomateriales como promotor de la creatividad científica en estudiantes de media secundaria. Universidad de los Andes.

* Rubiano, O. (2013). Construcción de una unidad didáctica para la enseñanza de los conceptos y términos más usados en nanociencia a través de indagación y la investigación [tesis de grado, MECEN].

* Serena, P. A. (2013, noviembre). Acercando la nanotecnología a la sociedad: la exposición "Un paseo por el nanomundo". Revista Digital Universitaria, 14(4). http:// www.revista.unam.mx/vol.14/num4/art29/

* Serena Domingo, Pedro Amalio. (2002): (s. f.) Nanociencia y nanotecnología: Aspectos generales. Encuentros Multidisciplinares, 12, 1-12. http://hdl.handle. net/10486/680428

* Torres, D. y Cabezas, A. (s. f.). Los blogs como nuevo medio de comunicación científica. http://eprints.rclis.org/11515/1/Torres-Salinas,_Daniel_y_Cabezas-Clavijo,_ Alvaro._Los_blogs_como_nuevo_medio_de_comunicacion_cientifica.pdf

* Torres, L. y Duarte, A. (2018). Docente-Nano: una alternativa para la divulgación del concepto de nanomateriales en la educación media [tesis de maestría, Universidad Nacional de Colombia].

* Tutor-SánchezJ. D. (2013, noviembre). Formación en nanocienciay nanotecnología: un reto en Iberoamérica, Revista de Física, 46E. http://bdigital.unal.edu. co/38750/1/41984-192810-1-PB.pdf

\section{Cómo CITAR ESTE ARTículo}

* Torres Romero, Lady Johana y Ariza Traslaviña, Leidy Bibiana. (2020, noviembre-diciembre). Diseño de un blog para la divulgación de nanociencia y nanotecnología. Revista Digital Universitaria (RDU), 21(6). Dol: http://doi.org/10.22201/ cuaieed.16076079e.2020.21.6.10 